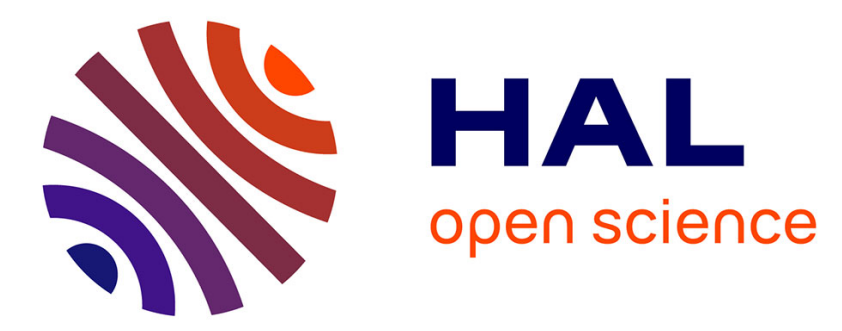

\title{
Time-domain interferometry for direct electric-field reconstruction by use of an acousto-optic programmable filter and a two-photon detector
}

Antoine Monmayrant, Manuel Joffre, Thomas Oksenhendler, R. Herzog, D. Kaplan, Pierre Tournois

\section{To cite this version:}

Antoine Monmayrant, Manuel Joffre, Thomas Oksenhendler, R. Herzog, D. Kaplan, et al.. Timedomain interferometry for direct electric-field reconstruction by use of an acousto-optic programmable filter and a two-photon detector. Optics Letters, 2003, 28 (4), pp.278-280. 10.1364/OL.28.000278 . hal-00836882

\section{HAL Id: hal-00836882}

https://hal-polytechnique.archives-ouvertes.fr/hal-00836882

Submitted on 16 May 2014

HAL is a multi-disciplinary open access archive for the deposit and dissemination of scientific research documents, whether they are published or not. The documents may come from teaching and research institutions in France or abroad, or from public or private research centers.
L'archive ouverte pluridisciplinaire HAL, est destinée au dépôt et à la diffusion de documents scientifiques de niveau recherche, publiés ou non, émanant des établissements d'enseignement et de recherche français ou étrangers, des laboratoires publics ou privés. 


\title{
Time-domain interferometry for direct electric-field reconstruction by use of an acousto-optic programmable filter and a two-photon detector
}

\author{
A. Monmayrant and M. Joffre \\ Laboratoire d'Optique et Biosciences, Centre National de la Recherche Scientifique, Unité Mixte de Recherche 7645, \\ Institut National de la Santé et de la Recherche Médicale U 451, Ecole Polytechnique, \\ Ecole Nationale Supérieure des Techniques Avancées, 91128 Palaiseau Cedex, France
}

T. Oksenhendler, R. Herzog, D. Kaplan, and P. Tournois

Fastlite, Xtec, Ecole Polytechnique, 91128 Palaiseau Cedex, France

Received July 16, 2002

\begin{abstract}
We introduce a new approach to the characterization of femtosecond optical pulses based on a remarkably simple setup combining a two-photon detector and a pulse shaper consisting of a longitudinal acousto-optic programmable filter. The operation of this setup is demonstrated through the use of a new version of spectral phase interferometry for direct electric-field reconstruction based on time-domain instead of on frequencydomain interferometry. (C) 2003 Optical Society of America
\end{abstract}

OCIS codes: $320.7100,320.5540,120.5050$.

The important problem of measuring the electric field $E(t)=\epsilon(t)+\epsilon^{*}(t)$ associated with an ultrashort pulse that is equivalent to the determination of the pulse's spectral amplitude $|\epsilon(\omega)|$ and phase $\varphi(\omega)$ has given rise to a great variety of experimental techniques. $^{1-3}$ In most such techniques, the input pulse is sent through a three-stage setup: first, a linear optical setup, e.g., a Michelson interferometer; second, a material inducing either second- or thirdorder nonlinear effects; and third, a time-integrating detection apparatus. ${ }^{1}$ An important simplification of this approach was achieved a few years ago by merger of the last two stages through the use of two-photon detectors that perform both the required optical nonlinearity and the detection. ${ }^{4-6}$ However, the first stage remains a complicated arrangement of discrete optical components that usually make the optical alignment of the measurement device quite critical. In this Letter we demonstrate a further simplification in which the entire first stage is replaced with a pulse shaper, as shown in Fig. 1. Indeed, despite its usual complexity, the first stage can be completely characterized by its linear response, a complex transfer function $R(\omega)$ in the case of collinear geometry. For example, the Michelson interferometer used in secondorder interferometric autocorrelators corresponds to $R(\omega)=[1+\exp (i \omega \tau)] / 2$. This transfer function can easily be achieved by use of a pulse shaper that permits complete amplitude and phase control such as the Dazzler, which is an acousto-optic programmable dispersive filter consisting of a 2.5 -cm-long $\mathrm{TeO}_{2}$ crys$\mathrm{tal}^{7,8}$ The use of this pulse shaper thus results in a dramatic reduction in size of the phase-measurement device.

To achieve complete phase characterization of the incident pulses by using our pulse shaper and a two-photon detector, we developed a variant of the homodyne optical technique for spectral phase interferometry for direct electric-field reconstruction (HOT SPIDER). ${ }^{9}$ This new version of the HOT SPIDER relies on time-domain instead of frequency-domain interferometry and thus makes the use of a grating spectrometer unnecessary. Our technique, hereafter referred to as time-domain HOT SPIDER, is based on the fact that the Fourier transform of a second-order interferometric autocorrelation trace yields the spectrum of the frequency-doubled incident pulse. ${ }^{10} \mathrm{~A}$ schematic of time-domain HOT SPIDER is shown in Fig. 2. Similarly to HOT SPIDER, time-domain HOT SPIDER relies on a two-step measurement in which each step involves the nonlinear mixing (here in the two-photon detector) of a sequence of three pulses: the pulse to be measured, $\epsilon(t)$, referred to as the unknown

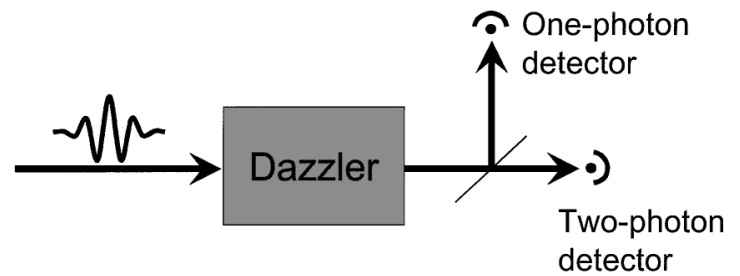

Fig. 1. Experimental setup for measuring an ultrashort pulse by use of a pulse shaper instead of an assembly of discrete optical components. The incident pulse is sent through the pulse shaper, here a Dazzler device, and detected with both a one-photon and a two-photon detector. If the pulse shaper generates two replicas of the incident pulse, this setup is equivalent to second-order interferometric autocorrelator. If the pulse shaper generates the sequence of pulses shown in Fig. 2, the setup is an implementation of time-domain HOT SPIDER. The one-photon detector is not absolutely required but makes possible the efficient measurement of the power spectrum through Fourier-transform spectroscopy. 


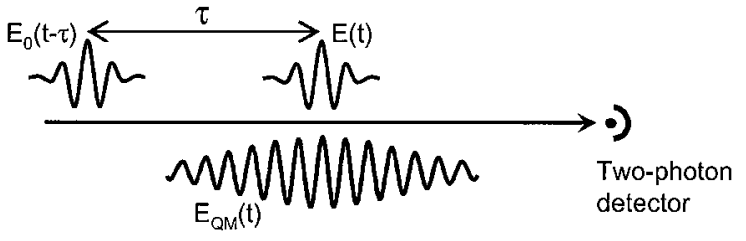

Fig. 2. Time-domain HOT SPIDER. The second-order nonlinear signal is recorded by a two-photon timeintegrating detector as a function of time delay $\tau$ between a reference pulse, $E_{0}$, and a superposition of two pulses: the pulse to be measured, $E$, and a quasimonochromatic pulse, $E_{\mathrm{QM}}$.

pulse; a quasi-monochromatic pulse, $\epsilon_{\mathrm{QM}, j}(t)$, of center frequency $\omega_{j}$; and a reference pulse, $\epsilon_{0}(t)$. In what follows, we designate by $\epsilon_{0}^{(2)}(t)=\epsilon_{0}(t)^{2}$ the squared reference pulse and by $\epsilon_{j}^{(2)}(t)=\epsilon(t) \epsilon_{\mathrm{QM}, j}(t)$ the cross term that results from nonlinear mixing between the unknown and the quasi-monochromatic fields. Assuming that the latter field is long enough, $\epsilon_{j}^{(2)}$ is simply a frequency-shifted replica of the unknown field, $\epsilon_{j}^{(2)}(\omega) \propto \epsilon\left(\omega-\omega_{j}\right)$, and its spectral phase is $\varphi\left(\omega-\omega_{j}\right)$. In the conventional HOT SPIDER, Fourier-transform spectral interferometry yields the quantity $\epsilon_{0}^{(2) *}(\omega) \epsilon_{j}^{(2)}(\omega)$, whose phase is $\varphi\left(\omega-\omega_{j}\right)-\varphi_{0}^{(2)}(\omega)$, where $\varphi_{0}^{(2)}(\omega)$ is the spectral phase of $\epsilon_{0}^{(2)}(\omega)$. The two steps mentioned above differ in the value of the center frequency of the quasi-monochromatic field, $\omega_{j}=\omega_{1}$ or $\omega_{j}=\omega_{2}$, with $\omega_{2}=\omega_{1}+\Omega$. The difference between the two measurements yields $\varphi\left(\omega-\omega_{2}\right)-\varphi\left(\omega-\omega_{1}\right) \approx \Omega \mathrm{d} \varphi / \mathrm{d} \omega$, from which the spectral phase can be deduced from concatenation or integration. ${ }^{9}$ The exact spectral phase of the reference pulse plays no role in the measurement and does not need to be known, which means that the reference pulse can be, for example, a replica of the unknown pulse itself. Note that in conventional SPIDER techniques the quasi-monochromatic field is usually replaced with a chirped pulse, which one can conveniently obtain by stretching the incident pulse.

There are two important differences between HOT SPIDER and time-domain HOT SPIDER: First, whereas in HOT SPIDER a grating spectrometer records the spectral interference between $\epsilon_{0}^{(2)}(\omega)$ and $\epsilon_{j}^{(2)}(\omega)$, time-domain HOT SPIDER relies on single-channel detection, which makes possible the use of the two-photon detector shown in Fig. 2. Second, time delay $\tau$ between the reference pulse and superposition $\epsilon(t)+\epsilon_{\mathrm{QM}, j}(t)$ is not maintained constant but needs to be scanned to record the second-order interferometric correlation:

$$
S_{j}^{(2)}(\tau)=\int_{-\infty}^{+\infty}\left[E_{0}(t-\tau)+E(t)+E_{\mathrm{QM}, j}(t)\right]^{4} \mathrm{~d} t .
$$

The Fourier transform of this quantity contains peaks centered at frequencies $0, \pm \omega_{0}$, and $\pm 2 \omega_{0}$, where $\omega_{0}$ is the pulse center frequency. By selecting in Eq. (1) the terms that correspond only to the peak at $+2 \omega_{0}$, we obtain the correlation product:

$$
\begin{aligned}
S_{j, 2 \omega}^{(2)}(\tau) & =\int_{-\infty}^{+\infty} \epsilon_{0}^{* 2}(t-\tau)\left[\epsilon(t)+\epsilon_{\mathrm{QM}, j}(t)\right]^{2} \mathrm{~d} t \\
& =\left(\epsilon_{0}^{(2) *}(-t) \otimes\left\{\left[\epsilon(t)+\epsilon_{\mathrm{QM}, j}(t)\right]^{2}\right\}\right)(\tau),
\end{aligned}
$$

where $\otimes$ stands for convolution. To extract the cross term $\epsilon(t) \epsilon_{\mathrm{QM}, j}(t)$ from the second-order interferometric correlation we chose to perform a sequence of two measurements in which the quasi-monochromatic field has been phase shifted by $\pi$. This phase shift does not change the terms that correspond to frequency doubling of each pulse while it changes the sign of the cross term. Therefore the difference between the two measurements yields the cross term $\epsilon(t) \epsilon_{\mathrm{QM}, j}(t)$. Finally, assuming that we have successfully selected the relevant term in Eq. (2), we obtain $\epsilon_{0}^{(2) *}(-t) \otimes \epsilon_{j}^{(2)}(t)$, whose Fourier transform yields $\varphi\left(\omega-\omega_{j}\right)-\varphi_{0}^{(2)}(\omega)$, as in conventional (frequency-domain) HOT SPIDER. The subsequent numerical treatment is exactly identical to that of the conventional technique.

As was mentioned above, time-domain HOT SPIDER is implemented here by use of the general approach of Fig. 1. The three fields shown in Fig. 2 are generated by superposition of three appropriate acoustic waves in the $\mathrm{TeO}_{2}$ crystal. In the linear acousto-optic diffraction regime, doing so results in diffracting from the crystal the required sum of the three electric fields. ${ }^{11}$ The scanning of the time delay is simply achieved by scanning of the time delay between two acoustic waveforms. Additionally, the generation of the $\pi$ phase shift mentioned above is straightforward, because it corresponds simply to changing the sign of the acoustic waveform. We thus obtain $S_{j, 2 \omega, \pm}^{(2)}(\tau)=\left(\epsilon_{0}^{(2) *}(-t) \otimes\left\{\left[\epsilon(t) \pm \epsilon_{\mathrm{QM}, j}(t)\right]^{2}\right\}\right)(\tau)$. The difference $S_{j, 2 \omega,+}^{(2)}(\tau)-S_{j, 2 \omega,-}^{(2)}(\tau)=4 \epsilon_{0}^{(2) *}(-t) \otimes \epsilon_{j}^{(2)}(t)$ then provides the information required for the phase retrieval. As shown in Fig. 1, we also collect a small part of the shaped beam, using a beam splitter, and measure the corresponding energy by using a

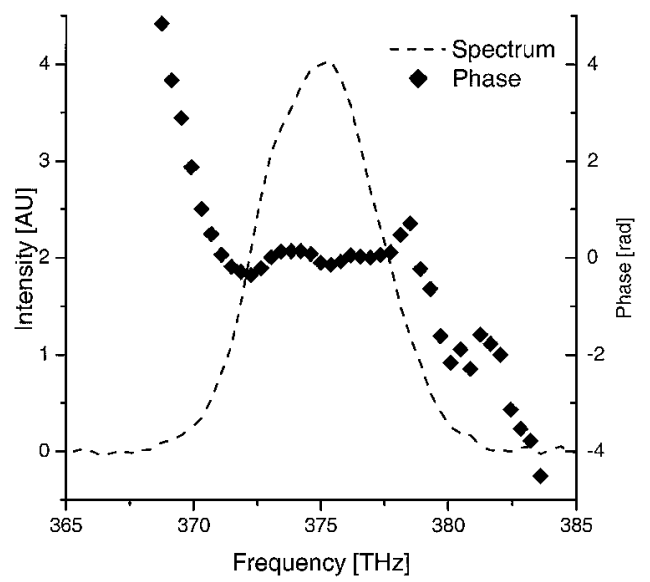

Fig. 3. Intensity (dashed curve) and phase retrieved for the pulses delivered by the pulse shaper by use of the time-domain HOT SPIDER procedure described in the text. 


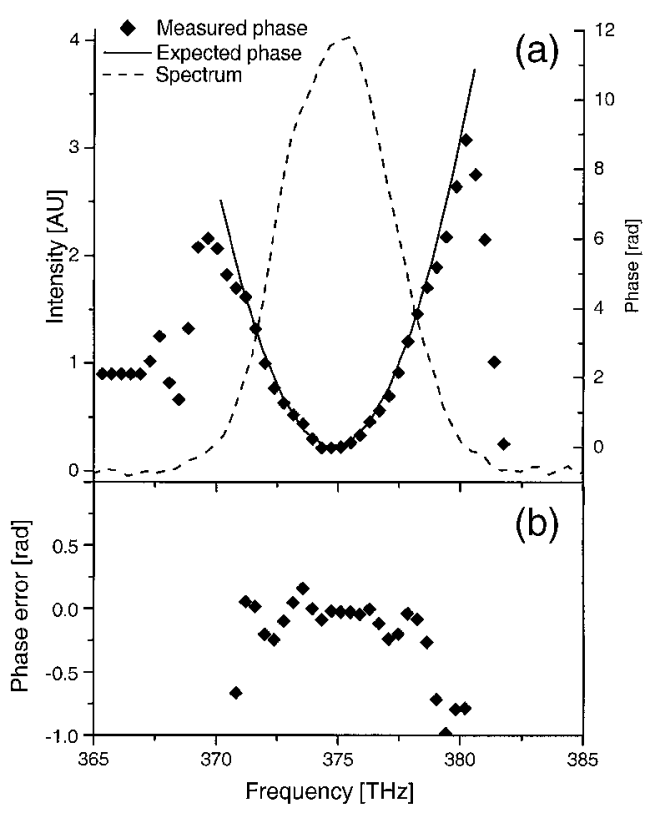

Fig. 4. (a) Diamonds, difference in spectral phase between two time-domain HOT SPIDER measurements that differ by a known amount of second-order phase $\left(8300 \mathrm{fs}^{2}\right)$. The expected spectral-phase difference and the spectral intensity (spectrum) are also shown. (b) Phase error computed by subtraction of the theoretical phase from the measured value.

one-photon detector. This measurement provides the power spectrum of the pulse and that of the quasi-monochromatic filtered pulse.

Figure 3 shows the spectral amplitude and phase retrieved for the pulses generated by a Hurricane $1-\mathrm{kHz}$ Ti:sapphire amplifier (Spectra-Physics) after transmission through the pulse shaper, which was programmed to compensate for its own linear dispersion. We verified that the spectrum obtained with the one-photon detector was identical to the frequency-shifted spectrum obtained from the two-photon measurement, although the signal-to-noise ratio was better in the former case. We then used the pulse shaper to add a known amount of second-order spectral phase to the incident beam and repeated the time-domain HOT SPIDER measurement.

Figure 4(a) shows the spectral-phase difference between the two measurements. To show the sensitivity of the measurement, we plot in Fig. 4(b) the difference between the measured and the theoretical values, which shows a phase error of only a few tenths of a radian for frequencies at which the spectral amplitude takes nonnegligible values. The good agreement between the measured phase and the expected value thus demonstrates the validity of our technique.

To summarize, we have demonstrated a new approach to pulse characterization based on the combination of a pulse shaper and a two-photon detector. This remarkably compact setup makes possible the implementation of many different pulse characterization techniques simply by programming the required waveform set in the acousto-optic programmable filter. Furthermore, we have introduced a new version of HOT SPIDER that relies on time-domain instead of frequency-domain interferometry. This time-domain HOT SPIDER technique is particularly suited to our pulse-shaper approach, as we demonstrated through the measurement of the spectral phase of $800-\mathrm{nm}$ femtosecond pulses. Our experiment also demonstrates that the unique ability of the longitudinal acousto-optic programmable filters to produce true $\pi$ phase shifts can be used to extract relevant terms even in a collinear geometry.

We thank Nadia Belabas, James Fraser, and Cathie Ventalon for fruitful discussions. M. Joffre's e-mail address is manuel.joffre@polytechnique.fr.

\section{References}

1. I. A. Walmsley and V. Wong, J. Opt. Soc. Am. B 13, 2453 (1996).

2. R. Trebino, K. DeLong, D. Fittinghoff, J. Sweester, M. Krumbügel, B. Richman, and D. Kane, Rev. Sci. Instrum. 68, 3277 (1997).

3. C. Dorrer and M. Joffre, C. R. Acad. Sci. Paris 2, 1415 (2001).

4. D. T. Reid, M. Padgett, C. McGowan, W. E. Sleat, and W. Sibbett, Opt. Lett. 22, 233 (1997).

5. W. Rudolph, M. Sheik-Bahae, A. Bernstein, and L. F. Lester, Opt. Lett. 22, 313 (1997).

6. J. K. Ranka, A. L. Gaeta, A. Baltuska, M. S. Pshenichnikov, and D. A. Wiersma, Opt. Lett. 22, 1344 (1997).

7. F. Verluise, V. Laude, Z. Cheng, Ch. Spielmann, and P. Tournois, Opt. Lett. 25, 575 (2000).

8. D. Kaplan and P. Tournois, J. Phys. IV France 12, Pr5-69 (2002).

9. C. Dorrer, P. Londero, and I. A. Walmsley, Opt. Lett. 26, 1510 (2001).

10. K. Naganuma, K. Mogi, and H. Yamada, IEEE J. Quantum Electron. 25, 1225 (1989).

11. The spectral bandwidth of our pulse shaper is limited to $100 \mathrm{THz}$ because of the electronics driving the acousto-optic transducer. This value is much greater than the $10-\mathrm{THz}$ bandwidth of our laser pulses and is therefore not detrimental to the measurement because the bandwidth is exactly centered on the laser's center frequency. 